\title{
Construção e Fidedignidade Teste-Reteste de Escalas de Silhuetas Brasileiras para Adultos e Crianças ${ }^{1}$
}

\author{
Idalina Shiraishi Kakeshita \\ Ana Idalina Paiva Silva \\ Daniela Perocco Zanatta \\ Sebastião Sousa Almeida ${ }^{2}$ \\ Universidade de São Paulo (Ribeirão Preto)
}

\begin{abstract}
RESUMO - O objetivo deste trabalho foi desenvolver escalas de silhuetas para crianças e adultos brasileiros de ambos os sexos e iniciar a avaliação de suas qualidades psicométricas. Foram fotografados adultos e crianças com Índice de Massa Corporal (IMC) previamente conhecidos, desenhadas as silhuetas e construídas as escalas por computação gráfica. Para avaliação da fidedignidade, procedeu-se ao teste-reteste com intervalo de um mês. As escalas foram aplicadas e reaplicadas a 90 adultos (18 a 60 anos) e 69 crianças ( 7 a 12 anos). As escalas apresentaram coeficientes de correlação entre teste e reteste positivos e significativos para o IMC real e IMC percebido como atual por adultos $(r=0,84 ; p<0,01)$ e crianças $(r=0,61, p<0,01)$. Concluiu-se que as escalas desenvolvidas constituem instrumentos apropriados à aplicação clínica e epidemiológica para avaliar a percepção da imagem corporal de crianças e adultos brasileiros.
\end{abstract}

Palavras-chave: imagem corporal; escalas de silhuetas; fidedignidade.

\section{A Figure Rating Scales for Brazilian Adults and Children: Development and Test-Retest Reliability}

\begin{abstract}
The aim of this work was to develop figure rating scales to evaluate the body image of Brazilian adults and children. Adults and children with known Body Mass Index (BMI) were photographed and had their silhouettes drawn. Based on that, the scales were made by computer graphics. To evaluate the reliability of the scales, test-retest was applied with a gap of one month. Participants included 90 adults (18-60 years old) and 69 children (7-12 years old) of both genders. The instruments presented positive and significant correlations between test and retest for the actual BMI and those perceived as current by adults $(r=0,84 ; p<0,01)$ and children $(r=0,61, p<0,01)$. It was concluded that the developed scales represent useful instruments for clinical and epidemiological research involving body image perception of Brazilian adults and children.
\end{abstract}

Key words: body image; figure rating scale; reliability.

A imagem corporal constitui relevante tema associado às questões referentes ao peso corporal, aos transtornos alimentares e, consequentemente, à qualidade de vida através do bem estar proporcionado pela satisfação corporal (Cash, 2004; Leonhard \& Barry, 1998; Thompson, 1996). É reconhecido o papel da insatisfação com a própria imagem corporal nos distúrbios alimentares e na produção do estresse característico da vida moderna, outro importante fator sociocultural associado ao quadro de morbimortalidade atual (Cash, 2004; Madrigal \& cols., 2000; Ramirez \& Rosen, 2001; Smolak, 2004). Lynch, Heil, Elise e Havens (2008) demonstraram que a insatisfação corporal em adolescentes americanas era o mediador de maior significância entre o Índice de Massa Corporal (IMC) e o risco de transtorno alimentar e comportamentos alimentares de risco à saúde. Sanchez-Johnsen, Hogan, Wilkens e Fitzgibbon (2008)

1 Fonte de Financiamento: CNPq.

2 Endereço para correspondência: Laboratório de Nutrição e Comportamento do Departamento de Psicologia e Educação da Faculdade de Filosofia, Ciências e Letras de Ribeirão Preto. Av. dos Bandeirantes, 3900. Ribeirão Preto, SP. CEP 14040-901. E-mail: sebasalm@usp.br. estudaram as correlações entre peso corporal, depressão e insatisfação corporal como preditores de comportamentos alimentares inadequados. Esses autores obtiveram resultados mostrando que mulheres não obesas com alta discrepância da imagem corporal ou alto escore para depressão, apresentavam aumento do risco para bulimia nervosa e transtorno da compulsão alimentar.

Com o aumento da incidência dos distúrbios relacionados à obesidade e aos padrões inadequados de conduta alimentar em idades cada vez mais precoces (Ambrosi-Randic, 2000; Davison \& Birch, 2001; Dietz, 1998), cresce também a preocupação em investigar as variáveis associadas, os fatores desencadeantes e as possibilidades de intervenção preventiva em relação a esses distúrbios ainda na infância (Button, Sonuga-Barke, Davies \& Thompson, 1996; Pinheiro \& Giugliani, 2006; Ricciardelli \& McCabe, 2001; Smolak, 2004).

Emergem questões relativas à influência dos fatores ambientais e sócio-culturais na insatisfação com a imagem corporal desde a infância, tais como: a idade a partir da qual essa influência é crítica, e quando a internalização dos modelos de corpo ideal passam a interferir na construção da identidade e da auto-imagem, e a gerar preocupações com 
o peso e a forma corporal (Hermes \& Keel, 2003; Sands \& Wardle, 2003, Smolak, 2004; Williamson \& Delin 2001). Skemp-Arlt, Rees, Mikat e Seebach (2006) encontraram que $50,6 \%$ de 261 crianças pré-adolescentes estudadas estavam insatisfeitas com o tamanho de seu corpo atual e 41,8\% desejavam ser mais esbeltas.

Diversos instrumentos para o estudo da percepção da imagem corporal têm sido desenvolvidos conforme o componente do constructo a ser focado (Cash \& Grasso, 2005; Hildebrandt \& Resnick, 2006; Williamson \& cols., 2000,). Um método de avaliação comumente utilizado emprega escalas de figuras de silhuetas que variam desde a mais esbelta até a mais larga. Cada participante é convidado a escolher a figura que melhor o representa e a que gostaria de ter ou que julga ser o ideal. A satisfação ou insatisfação com a imagem corporal é avaliada conforme as discrepâncias entre as figuras selecionadas (Fingeret, Gleaves \& Pearson, 2004).

As escalas de figura de silhuetas possuem inúmeras vantagens: constituem instrumento simples, de fácil aplicação e não exigem equipamentos sofisticados. Suas imagens visuais, menos abstratas, não requerem grande diversidade de vocabulário ou fluência verbal, o que as torna especialmente indicadas na avaliação da percepção da imagem corporal, principalmente de crianças. Entretanto, no caso dessas últimas, cabe ressaltar a importância de adequar as figuras no sentido de representarem silhuetas infantis e as divergências dos resultados quanto a acurácia dessa técnica para avaliar medidas da percepção e insatisfação com a imagem corporal de crianças menores de 7 anos (Truby \& Paxton 2002; Williamson \& Delin, 2001).

As escalas de silhuetas existentes, tanto para adultos quanto para crianças, variam quanto ao número de figuras, forma das silhuetas desenhadas, tamanho e forma de apresentação das escalas. A metodologia aplicada também varia, desde as características da amostra, os procedimentos e tratamento dos dados, até a análise e interpretação dos resultados (Stunkard, 2000; Tehard, Van Liere, Com Nogué \& Clavel-Chapelon, 2002; Thompson, 1996; Wertheim, Paxton \& Tilgner, 2004).

Nesse sentido, além dos trabalhos já citados, têm sido conduzidos estudos que atestam a validade preditiva da escala de silhuetas na avaliação da imagem corporal, em diferentes contextos. E cresce o interesse pela pesquisa dos fatores ambientais implicados na complexa temática da imagem corporal. Bessenoff e Snow (2006) examinaram como as mulheres universitárias percebem o padrão de corpo cultural e socialmente estabelecido, a influência da internalização desse padrão como ideal e sentimentos auto-depreciativos. O estudo de Berg e cols. (2007) avaliou o papel do corpo veiculado pela mídia como mediador entre a auto-estima, a depressão, o ambiente dietético dos pais, a dieta de amigos, a exposição às mensagens da TV e revistas, as preocupações com o peso corporal e o IMC, e a insatisfação corporal, em homens e mulheres. McCabe, Ricciardelli, Sitaram e Mikhail (2006) avaliaram os fatores biopsicossociais relacionados à acurácia na estimação do próprio tamanho corporal e a insatisfação corporal de adultos. Eles incluíram como variáveis o IMC, a depressão, mensagens dos pares e a mídia sobre o corpo. Legenbauer, Rühl e Vocks (2008) investigaram a influência da exposição à mídia na imagem corporal em pacientes com transtorno alimentar e um grupo controle sem transtorno.

Reconhecida a importância da devida adaptação de quaisquer instrumentos de avaliação psicológica ao contexto sócio cultural no âmbito de sua aplicação (Anastasi \& Urbina, 2000), ressalta-se o fato de não existirem no Brasil instrumentos próprios ou metodologia testada para a avaliação da imagem corporal nem de adultos nem de crianças. Portanto, o objetivo deste trabalho foi desenvolver escalas de silhuetas adaptadas ao contexto sócio-econômico e cultural brasileiro, para adultos e crianças, e testar sua fidedignidade como etapa inicial do processo de validação e padronização das mesmas.

\section{Método}

\section{A construção das escalas}

Estabeleceu-se a quantidade de figuras que comporiam cada escala, em termos do intervalo de abrangência e representação do IMC para adultos e crianças. Foram previamente definidos o valor constante de incremento e os valores médios de IMC correspondentes a cada figura. Considerou-se a altura média brasileira de 1,72 $\mathrm{m}$ para homens e 1,65 m para mulheres; e, no caso das crianças, fixou-se a média de altura da população infantil brasileira, aos 10 anos de idade, em 140,15 cm para meninos e $141,25 \mathrm{~cm}$ para meninas (Monteiro, Benício \& Gouveia, 2000).

Foram fotografadas crianças e adultos, com IMC correspondentes às médias dos intervalos estabelecidos para as figuras da sequência das escalas. As fotografias foram realizadas individualmente por profissional habilitado, em estúdio fotográfico próprio, em fundo branco, com os participantes na mesma postura (de frente, com as pernas ligeiramente afastadas, homens com as mãos nos quadris e mulheres com uma das mãos nos quadris e a outra ao longo do corpo). Desenhadas as silhuetas a partir dessas fotos, as escalas foram construídas por computação gráfica também por profissional habilitado da área. Para as escalas de crianças, os traços das figuras foram adequados a partir do modelo adulto, para melhor caracterizarem a faixa etária infantil.

Para garantir as qualidades psicométricas do instrumento, conforme recomendações de Gardner, Friedman e Jackson (1998) e Gardner, Stark, Jackson e Friedman (1999), foram considerados os seguintes aspectos na sua construção: (1) incremento constante nos intervalos de IMC estabelecidos para a sequência das figuras; (2) número suficiente de figuras para abranger o máximo de possibilidades; (3) magnitude da diferença constante entre os desenhos das figuras adjacentes; (4) eliminação de possíveis elementos de distração da percepção visual; (5) apresentação das figuras em cartões individuais, identificadas numericamente no verso.

\section{Participantes}

A amostra total para o teste das qualidades psicométricas das escalas construídas foi constituída por 280 adultos e 160 
crianças, voluntários, de diferentes classes sócio-demográficas, níveis de escolaridade diversos, no caso de adultos, e com IMC representativo de todos os intervalos das escalas. Por se tratar de método psicofísico clássico, amplamente validado na literatura de percepção, os resultados obtidos são pouco discrepantes entre participantes, permitindo a utilização de uma amostra relativamente pequena.

A amostra para o estudo procurou acompanhar a distribuição da prevalência de eutrofia, déficit de peso, excesso de peso e obesidade na população brasileira, conforme levantado no período de 2002-2003 pela Pesquisa de Orçamentos Familiares (POF) realizada pelo Instituto Brasileiro de Geografia e Estatística (IBGE), e divulgados pela Coordenação Geral da Política de Alimentação e Nutrição do Ministério da Saúde (2005). Procurou-se estabelecer um número mínimo de participantes para que a amostra contivesse representantes correspondentes a todos os intervalos de IMC da escala construída, e que fosse viável na prática para os pesquisadores envolvidos e possibilitasse o alcance dos objetivos propostos pela pesquisa.

Para a avaliação da fidedignidade das escalas submeteram-se ao teste-reteste um total de 90 adultos (46 mulheres e 44 homens) e 69 crianças (43 meninas e 26 meninos), conforme indicado na Tabela 1 , representantes de todos os intervalos de IMC correspondentes a cada uma das figuras de silhuetas das escalas, e das demais variáveis (sexo, idade, extrato sócio-econômico e escolaridade). A amostra contou com representantes de todos os níveis de escolaridade (primeiro, segundo e terceiro graus), assim como representantes de todas as classes sócio-econômicas (A a D) de acordo com o Critério de Classificação Econômica Brasil, da Associação Brasileira de Empresas de Pesquisa - ABEP (2003).

\section{Procedimento}

No caso de adultos, exposta a natureza da pesquisa e assinado o Termo de Consentimento, foram anotados seus dados pessoais e de classificação sócio-demográfica. A seguir, apresentada a escala em série ordenada ascendente (primeiro a de seu próprio gênero), cada adulto foi solicitado a escolher, na seguinte sequência, "a figura que melhor representasse seu tamanho atual", "a figura que representasse o tamanho que gostaria de ter" e "a figura que consideraria o tamanho ideal para o próprio gênero em geral". Os cartões eram recolhidos e, apresentados aqueles do gênero oposto, solicitava-se a escolha da "figura que melhor representaria o ideal para aquele gênero em geral". Posteriormente, cada sujeito tinha seu peso aferido em balança eletrônica portátil (Kratos-Kas) sem calçados e objetos ou vestuário pesados. A altura foi obtida por antropômetro portátil junto à parede sem rodapés, com a pessoa encostada à superfície, ereta, de modo que os olhos e as orelhas traçassem linhas horizontais paralelas. Desta forma, as normas para aferição de peso e estatura foram obedecidas.

Para o teste das escalas infantis, foram feitos contatos com escolas, obtidas as autorizações e enviados aos pais os termos de consentimento da participação da criança e o questionário de classificação sócio-econômica a serem preenchidos e assinados. Obtido o consentimento dos pais e das crianças,

Tabela 1. Caracterização demográfica descritiva ( $\mathrm{IC}=95 \%$ ) das amostras adulta e infantil, por gênero.

\begin{tabular}{|c|c|c|c|c|}
\hline \multirow{2}{*}{ AMOSTRA } & \multicolumn{2}{|c|}{ ADULTOS } & \multicolumn{2}{|c|}{ CRIANÇAS } \\
\hline & Feminina & Masculina & Feminina & Masculina \\
\hline $\mathbf{N}$ & 46 & 44 & 43 & 26 \\
\hline \multicolumn{5}{|l|}{ Idade } \\
\hline Média & 36,35 & 31,29 & 9,81 & 9,23 \\
\hline EPM & 1,60 & 1,74 & 0,22 & 0,30 \\
\hline IC & $19-57$ & $18-59$ & $7-12$ & $7-12$ \\
\hline \multicolumn{5}{|l|}{ Peso (kg) } \\
\hline Média & 72,98 & 88,89 & 37,96 & 39,85 \\
\hline EPM & 3,46 & 3,86 & 1,60 & 2,38 \\
\hline IC & $33,10-129,40$ & $52,50-168,00$ & $21,65-60,60$ & $22,60-67,90$ \\
\hline \multicolumn{5}{|l|}{ Altura (m) } \\
\hline Média & 1,60 & 1,75 & 1,42 & 1,41 \\
\hline EPM & 0,01 & 0,01 & 0,02 & 0,02 \\
\hline IC & $1,47-1,78$ & $1,63-1,90$ & $1,19-1,65$ & $1,24-1,62$ \\
\hline \multicolumn{5}{|l|}{ IMC Real } \\
\hline Média & 28,41 & 28,77 & 18,54 & 19,80 \\
\hline EPM & 1,33 & 1,20 & 0,54 & 0,89 \\
\hline IC & $12,31-52,50$ & $17,42-52,43$ & $13,56-27,13$ & $13,91-30,33$ \\
\hline
\end{tabular}

EPM=Erro Padrão da Média; IC=Intervalo de Confiança. 

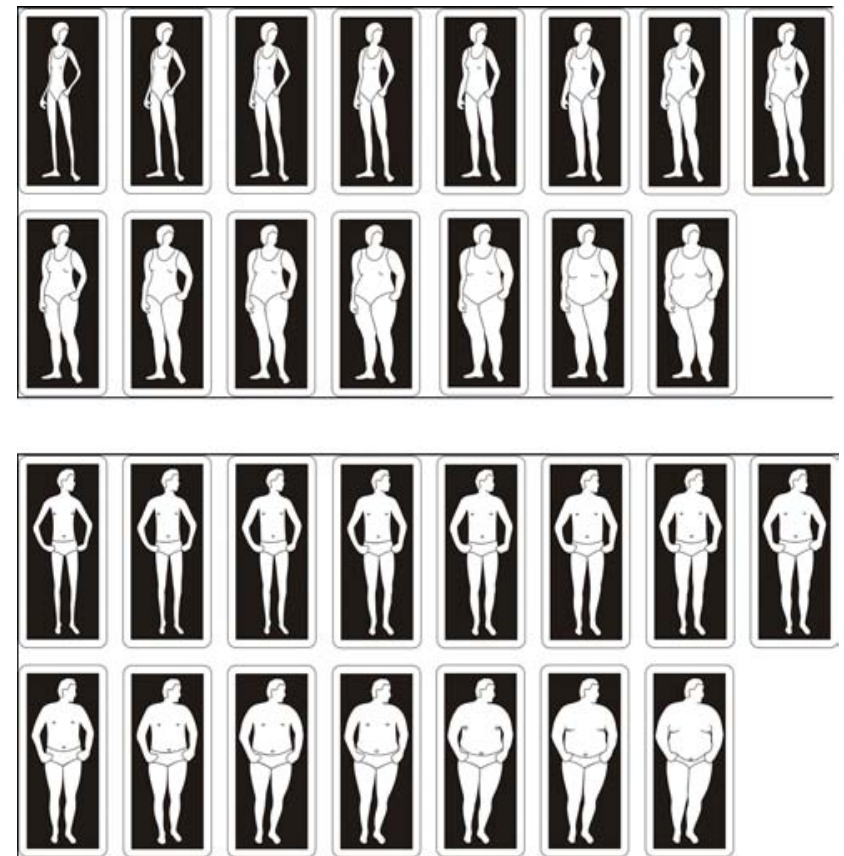

Figura 1. Escala de Silhuetas para adultos.

a coleta de dados ocorreu em datas pré-estabelecidas e em local próprio cedido pela escola. As crianças foram pesadas e medidas conforme as normas anteriormente descritas e com os mesmos instrumentos, e responderam às questões referentes à percepção da imagem corporal. Apresentada a escala de seu próprio gênero em ordem ascendente, foi-lhe perguntado "Qual figura representa o seu corpo atual?", "Qual figura representa o corpo que você gostaria de ter?" e "Qual figura representa o corpo ideal?".

Os procedimentos foram repetidos com intervalo de um mês, tanto para adultos quanto para crianças.

Este trabalho foi aprovado pelo Comitê de Ética em Pesquisa da Faculdade de Filosofia, Ciências e Letras de Ribeirão Preto da Universidade de São Paulo.

\section{Análise de dados}

Os dados foram tabulados e classificados por gênero. Foi calculado o IMC real e registrados os IMCs escolhidos em resposta às perguntas formuladas pelo experimentador, de acordo com o protocolo experimental. Após a estatística descritiva, realizou-se a correlação de Pearson e o teste $t$ pareado para a análise da fidedignidade teste-reteste. Para a análise estatística, utilizou-se o programa SPSS para Windows, versão 12.

\section{Resultados}

As escalas foram compostas por 15 cartões plastificados para adultos e 11 cartões para crianças, para cada gênero, com $12,5 \mathrm{~cm}$ de altura por $6,5 \mathrm{~cm}$ de largura, com a figura branca centralizada em fundo negro de $10,5 \mathrm{~cm}$ de altura por $4,5 \mathrm{~cm}$ de largura. Nas escalas de adultos, as médias de IMC
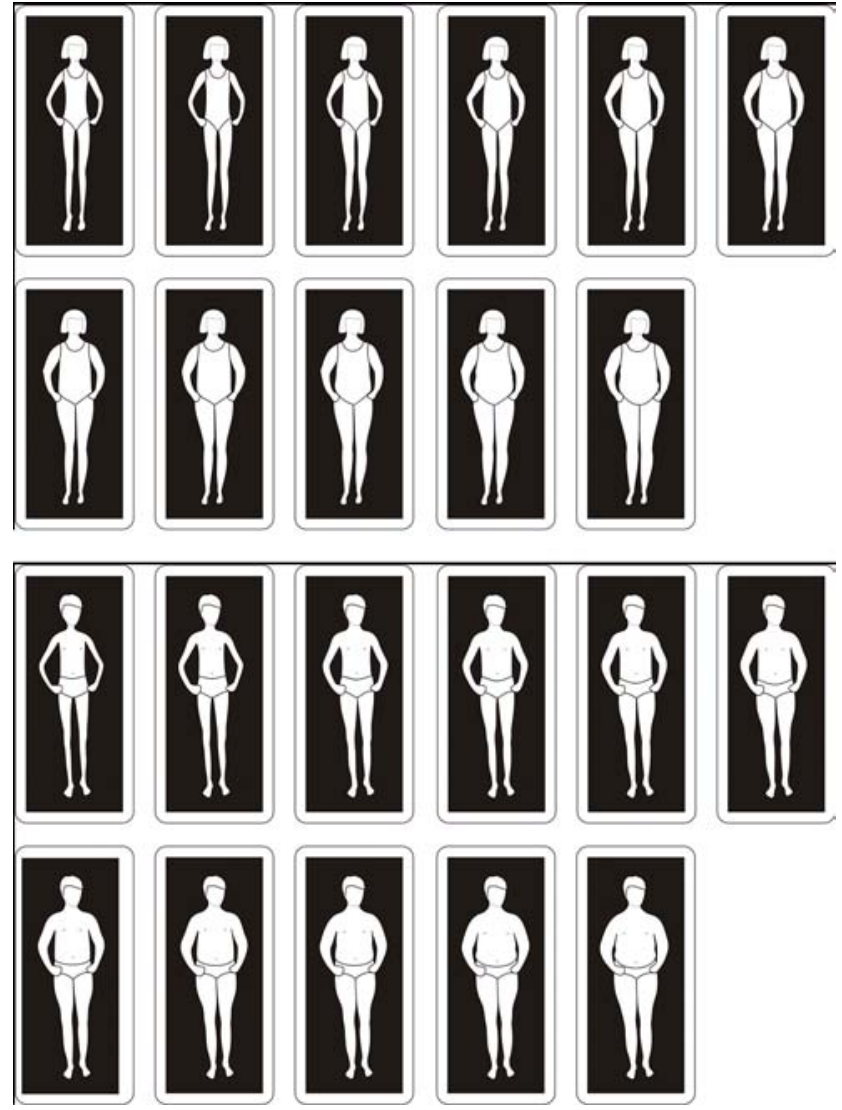

Figura 2. Escala de Silhuetas para crianças.

correspondentes a cada figura variaram de 12,5 a $47,5 \mathrm{~kg} /$ $\mathrm{m}^{2}$, com diferença constante de 2,5 pontos. Nas escalas de crianças, as médias de IMC correspondentes a cada figura variaram de 12 a $29 \mathrm{~kg} / \mathrm{m}^{2}$ (Figura 1 e Figura 2).

Conforme apresentado na Tabela 2, as escalas de silhuetas para adultos masculinos e femininos apresentaram correlações de Pearson positivas entre o IMC real e aquele reconhecido como atual $(r=0,84 ; p<0,01)$. As correlações para as silhuetas atuais e desejadas de mulheres e homens mostraram-se de acordo com aquelas esperadas em uma avaliação de fidedignidade, próximas a 0,80 ou 0,90 (Anastasi \& Urbina 2000). Apenas as correlações relativas às silhuetas apontadas pelos homens como ideais foram $0,53 \mathrm{e} 0,54$, sendo as outras correlações próximas ao mínimo recomendado de 0,70 (Thompson 2004). Observa-se também, que o teste $t$ pareado não mostrou diferença significativa entre os dados coletados no teste quando comparados aos dados coletados no reteste. Isso mostra que, além de uma correlação de Pearson positiva e significativa, o teste $t$ de Student para medidas pareadas também aponta para a fidedignidade do instrumento. Com relação à amostra total, observa-se que a maioria dos coeficientes de correlação se aproximam dos valores recomendados, ou seja, 0,70 ou mais.

Quanto à escala infantil, mostrada na Tabela 3, os dados são semelhantes àqueles descritos para as escalas de silhuetas dos adultos. Como esperado, a correlação entre o IMC da figura apontada como percepção atual e o IMC real das crianças mostrou-se positiva e significativa tanto para meninas $(r=0,57 ; \mathrm{p}<0,01)$ como meninos $(r=0,64 ; \mathrm{p}<0,01)$. Os 
Tabela 2. Média (erro padrão da média) e IC (95\%) do IMC Real e IMCs apontados como Atual, Desejado, Ideal feminino e Ideal masculino, seguidos pelos coeficientes de correlação de Pearson e teste $t$ pareado na avaliação da fidedignidade teste-reteste da escala para adultos.

\begin{tabular}{|c|c|c|c|}
\hline AMOSTRA & FEMININA & MASCULINA & TOTAL \\
\hline $\mathbf{N}$ & 46 & 44 & 90 \\
\hline IMC Real & $28,41(1,34)$ & $28,77(1,20)$ & $28,58(0,90)$ \\
\hline IC & $12,31-52,50$ & $17,42-52,43$ & $12,31-52,50$ \\
\hline $\begin{array}{l}\text { IMC Atual/Real } \\
\text { Correlação de Pearson }\end{array}$ & $0,84^{*}$ & $0,89 *$ & $0,84 *$ \\
\hline IMC Atual (Teste) & $31,63(1,26)$ & $29,55(1,61)$ & $30,61(1,02)$ \\
\hline IC & $12,50-47,50$ & $12,50-47,50$ & $12,50-47,50$ \\
\hline IMC Atual (Reteste) & $30,00(1,28)$ & $28,69(1,45)$ & $29,36(0,96)$ \\
\hline IC & $12,50-47,50$ & $17,50-47,50$ & $12,50-47,50$ \\
\hline Correlação de Pearson & $0,92 *$ & $0,93^{*}$ & $0,92 *$ \\
\hline Teste $t$ pareado & ns & ns & ns \\
\hline IMC Desejado (Teste) & $25,65(0,86)$ & $24,77(0,80)$ & $25,22(0,58)$ \\
\hline IC & $15,00-37,50$ & $17,50-37,50$ & $15,00-37,50$ \\
\hline IMC Desejado (Reteste) & $24,95(0,84)$ & $24,72(0,72)$ & $24,83(0,55)$ \\
\hline IC & $15,00-35,00$ & $17,50-37,50$ & $15,00-37,50$ \\
\hline Correlação de Pearson & $0,85^{*}$ & $0,70 *$ & $0,79 *$ \\
\hline Teste $t$ pareado & ns & ns & ns \\
\hline IMC Ideal $q$ (Teste) & $23,75(0,64)$ & $24,77(0,42)$ & $24,25(0,39)$ \\
\hline IC & $15,00-32,50$ & $20,00-32,50$ & $15,00-32,50$ \\
\hline IMC Ideal + (Reteste) & $23,42(0,63)$ & $25,28(0,41)$ & $24,33(0,39)$ \\
\hline IC & $15,00-32,50$ & $20,00-32,50$ & $15,00-32,50$ \\
\hline Correlação de Pearson & $0,71^{*}$ & $0,53 *$ & $0,67^{*}$ \\
\hline Teste $t$ pareado & ns & ns & ns \\
\hline IMC Ideal $\widehat{\jmath}$ (Teste) & $23,36(0,55)$ & $23,92(0,64)$ & $23,64(0,42)$ \\
\hline IC & $15,00-32,50$ & $12,50-35,00$ & $12,50-35,00$ \\
\hline IMC Ideal $\widehat{\jmath}$ (Reteste) & $23,10(0,53)$ & $23,86(0,54)$ & $23,47(0,38)$ \\
\hline IC & $15,00-32,50$ & $17,50-32,50$ & $15,00-32,50$ \\
\hline Correlação de Pearson & $0,66^{*}$ & $0,54^{*}$ & $0,60^{*}$ \\
\hline Teste $t$ pareado & ns & ns & ns \\
\hline
\end{tabular}

$\mathrm{IMC}=$ Índice de Massa Corporal; IC=Intervalo de Confiança; ns=não significativo; * $p<.01$.

IMCs para as avaliações das silhuetas atual, desejada e ideal também mostraram correlações positivas e significativas para ambos os sexos, o que se refletiu em valores próximos ao considerado ideal na análise da amostra total. Também, como nas escalas de silhuetas para adultos, o teste $t$ para medidas pareadas não mostrou diferenças significativas entre os dados coletados no teste e os dados coletados no reteste. Esses dados do teste $t$ reforçam aqueles da correlação de Pearson, apontando para a fidedignidade do instrumento construído para avaliar a imagem corporal de crianças.

\section{Discussão}

Muitos trabalhos com escalas de silhuetas não trazem as medidas das qualidades psicométricas do instrumento antes da sua aplicação, o que é criticado por diversos autores
(Gardner, Stark, Jackson \& Friedman, 1999; Smolak, 2004; Thompson, 2004; Thompson \& Gray, 1995). Thompson e Gray (1995) fizeram uma revisão das propriedades psicométricas de 22 escalas desenvolvidas entre 1959 e 1993, e encontraram dados de fidedignidade apenas para cinco dessas escalas e medidas de validade apenas para três delas. Thompson (2004), ao apontar 10 estratégias para refinar a avaliação de medidas de imagem corporal, incluiu a importância de se selecionar medidas com fidedignidade e validade estabelecidas e aceitáveis, como evidência de sua sensibilidade. Ainda, Gardner e cols. (1999) e Smolak (2004), em suas pesquisas na literatura sobre imagem corporal em crianças e adolescentes, referiram-se à insuficiência ou inexistência de dados psicométricos da maioria das escalas de silhuetas utilizadas nas pesquisas com crianças e adolescentes, entre outros problemas metodológicos. 
Tabela 3. Média (erro padrão da média) e IC (95\%) do IMC Real e IMCs apontados como Atual, Desejado e Ideal, seguidos pelos coeficientes de correlação de Pearson e teste $t$ pareado na avaliação da fidedignidade teste-reteste da escala infantil.

\begin{tabular}{|c|c|c|c|}
\hline AMOSTRA & FEMININA & MASCULINA & TOTAL \\
\hline $\mathbf{N}$ & 43 & 26 & 69 \\
\hline IMC Real & $18,54(0,54)$ & $19,80(0,89)$ & $19,02(0,48)$ \\
\hline IC & $13,56-27,13$ & $13,91-30,33$ & $13,56-30,33$ \\
\hline $\begin{array}{l}\text { IMC Atual/Real } \\
\text { Correlação de Pearson }\end{array}$ & $0,57^{*}$ & $0,64 *$ & $0,61^{*}$ \\
\hline IMC Atual (Teste) & $18,84(0,59)$ & $20,17(0,74)$ & $19,34(0,47)$ \\
\hline IC & $13,70-27,30$ & $13,70-27,30$ & $13,70-27,30$ \\
\hline IMC Atual (Reteste) & $18,17(0,60)$ & $19,52(0,74)$ & $18,68(0,47)$ \\
\hline IC & $12,00-29,00$ & $13,70-25,60$ & $12,00-29,00$ \\
\hline Correlação de Pearson & $0,73^{*}$ & $0,58^{*}$ & $0,68^{*}$ \\
\hline Teste $t$ pareado & ns & ns & ns \\
\hline IMC Desejado (Teste) & $16,03(0,50)$ & $17,43(0,54)$ & $16,56(0,38)$ \\
\hline IC & $12,00-3,90$ & $12,00-22,20$ & $12,00-23,90$ \\
\hline IMC Desejado (Reteste) & $16,35(0,50)$ & $17,62(0,47)$ & $16,83(0,36)$ \\
\hline IC & $12,00-27,30$ & $12,00-22,20$ & $12,00-27,30$ \\
\hline Correlação de Pearson & $0,67 *$ & $0,50 *$ & $0,64^{*}$ \\
\hline Teste $t$ pareado & ns & ns & ns \\
\hline IMC Ideal (Teste) & $17,06(0,41)$ & $17,88(0,57)$ & $17,37(0,34)$ \\
\hline IC & $12,00-27,30$ & $12,00-22,20$ & $12,00-27,30$ \\
\hline IMC Ideal (Reteste) & $17,06(0,41)$ & $17,49(0,59)$ & $17,17(0,34)$ \\
\hline IC & $12,00-27,30$ & $12,00-23,90$ & $12,00-23,90$ \\
\hline Correlação de Pearson & $0,67 *$ & $0,84^{*}$ & $0,74 *$ \\
\hline Teste $t$ pareado & ns & ns & ns \\
\hline
\end{tabular}

$\mathrm{IMC}=$ Índice de Massa Corporal; IC=Intervalo de Confiança; ns=não significativo; * $p<.01$.

A alta correlação positiva entre o IMC real e aquele percebido como atual $(r>0,84 ; p<0,01)$ evidencia a validade de construto da escala para adultos deste trabalho, como já demonstrado em outros estudos. Gardner e cols., (1999) desenvolveram três escalas diferentes para avaliar a percepção do tamanho corporal: uma visual analógica com duas figuras, uma com 13 figuras e uma metodologia por vídeo câmera e distorção de imagens digitalizadas. Esses autores encontraram coeficientes de correlação entre o tamanho corporal percebido como atual e o IMC real de 0,63 com a escala de duas figuras, 0,58 com a escala de 13 figuras, e 0,81 no método de vídeo distorção, ao nível de significância de 0,001, em uma amostra de 100 participantes. Nessa mesma pesquisa, a escala de nove figuras de Stunkard, Sorensen e Schulsinger (1983), mais comumente utilizada, apresentou um coeficiente de correlação de 0,67. Thompson e Gray (1995) conduziram o teste-reteste da escala de nove figuras que desenvolveram, com uma semana de intervalo, em uma amostra de 32 participantes, e obtiveram um coeficiente de fidedignidade de $0,78(p<0,05)$ para o tamanho corporal atual, e uma correlação de 0,59 entre a figura atual e o IMC autoinformado. Williamson e cols, (2000) desenvolveram uma escala de silhuetas para avaliação na obesidade e relataram coeficientes de fidedignidade teste-reteste de 0,77 para a silhueta atual, com duas semanas de intervalo, em uma amos- tra de 77 participantes. Wertheim, Paxton e Tilgner (2004) conduziram um estudo para avaliação da fidedignidade testereteste com intervalos diferentes e validade de construto da escala de silhuetas desenvolvida por Thompson e Gray em uma amostra de adolescentes. Eles encontraram valores entre 0,77 e 0,84 para a figura atual, e reforçaram a importância de se avaliar as qualidades psicométricas das escalas de silhuetas no contexto de sua aplicação, como constante das diretrizes da American Psychological Association (2000).

A correlação positiva e significativa obtida neste estudo também foi corroborada pelos resultados do teste $t$ pareado, que não indicou diferenças significativas entre o teste e o reteste.

A maioria das escalas desenvolvidas apresenta abrangência restrita, tanto no número de figuras quanto na magnitude do intervalo de IMC, contribuindo para um viés metodológico na medida em que limita as respostas (Gardner \& cols., 1998). Ao contrário dessas, o intervalo de abrangência das figuras das presentes escalas garante a inclusão de casos extremos de IMC, conferindo amplas possibilidades de utilização clínica e epidemiológica ao instrumento.

A escala infantil, em concordância com outros autores (Gardner, Stark, Friedman \& Jackson, 2000; Ricciardelli \& McCabe, 2001; Truby \& Paxton, 2002; Williamson \& Delin, 2001), também atendeu às recomendações de fidedignidade 
para as silhuetas apontadas como atuais, tanto para meninas quanto para meninos, mostrando coeficientes de correlação positivos e significativos e corroborados com os resultados do teste $t$ pareado, que não mostraram diferenças significativas entre os dados do teste e do reteste.

Esses resultados reforçaram a capacidade das crianças dessa faixa etária em identificar o próprio tamanho corporal, como mencionado pelos autores já citados, e vieram atender à necessidade de instrumentos adequados, devidamente testados, que deem suporte às pesquisas no campo da imagem corporal, em franca expansão (Ricciardelli \& McCabe, 2001; Smolak, 2004; Thompson, 2004).

Como etapa inicial do processo de adaptação de escalas de silhuetas para a população adulta e infantil brasileira, os resultados sugerem que os instrumentos foram apropriadamente construídos e, nesse contexto, tiveram sua fidedignidade teste-reteste demonstrada para os estudos que envolvam a percepção da imagem corporal.

\section{Referências}

American Psychological Association (2000). Report of the task force on test user qualifications. Washington: APA.

Associação Brasileira de Empresas de Pesquisa - ABEP (2003). Critério de Classificação Econômica Brasil. Retirado em 16/03/2007, de http://www.abep.org.br/codigosguias/ABEP_CCEB.pdf.

Ambrosi-Randic, N. (2000). Perception of current and ideal body size in preschool age children. Perceptual and Motor Skills, 90, 885-889.

Anastasi, A., \& Urbina, S. (2000). Testagem psicológica, Porto Alegre: Artes Médicas Sul.

Berg, P. van den, Paxton, S. J., Keery, H., Wall, M., Guo, J., \& Neumark-Sztainer, D. (2007). Body Image, 4, 257-268.

Bessenoff, G. R., \& Snow, D. (2006). Absorbing society's influence: Body image self-discrepancy and internalized shame. Sex Roles, 54, 727-731.

Button, E. J., Sonuga-Barke, E. J. S., Davies, J., \& Thompson, M. (1996). A prospective study of self-esteem in the prediction of eating problems in adolescent schoolgirls: Questionnaire findings. British Journal of Clinical Psychology, 35, 193-203.

Cash, T. F. (2004). Body image: Past, present, and future. Body Image, 1, 1-5.

Cash, T. F., \& Grasso, K. (2005). The norms and stability of new measures of the multidimensional body image construct. Body Image, 2, 199-203.

Dietz, W. H. (1998). Health consequences of obesity in youth: Children predictors of adult disease. Pediatrics, 101, 518-525.

Davison, K. K., \& Birch, L. L. (2001). Weight status, parent reaction, and self-concept in five-year-old girls. Pediatrics, 107, 46-53.

Fingeret, M. C., Gleaves, D. H., \& Pearson, C. A. (2004). On the methodology of body image assessment: The use of figural rating scales to evaluate body dissatisfaction and the ideal body standards of women. Body Image, 1, 207-212.

Gardner, R. M., Friedman, B. N., \& Jackson, N. A. (1998). Methodological concerns when using silhouettes to measure body image. Perceptual and Motor Skills, 86, 387-395.
Gardner, R. M., Stark, K., Jackson, N. A., \& Friedman, B. N. (1999). Development and validation of two new scales for assessment of body image. Perceptual and Motor Skills, 89, 981993.

Gardner, R. M., Stark, K., Friedman, B. N., \& Jackson, N. A. (2000). Predictors of eating disorder scores in children ages 6 through 14. A longitudinal study. Journal of Psychosomatic Research, 49, 199-205.

Hermes, S. F., \& Keel, P. K. (2003). The influence of puberty and ethnicity on awareness and internalization of the thin ideal. Internationl Journal of Eating Disorders, 33, 465-467.

Hildebrandt, T., \& Resnick, C. (2006). Evidence that ideal and attractive figures represent different constructs: A replication and extension of Fingeret, Gleaves, and Pearson (2004). Body Image, 3, 173-182.

Legenbauer, T., Rühl, I., \& Vocks, S. (2008). Influence of appearance-related TV commercials on body image state. Behavior Modification, 32(3), 352-371.

Leonhard, M. L., \& Barry, N. J. (1998). Body image and obesity: Effects of gender and weight on perceptual measures of body image. Addictive Behaviors, 23(1), 31-34.

Lynch, W. C., Heil, D. P., Wagner, E., \& Havens, M. D. (2008). Body dissatisfaction mediates the association between body mass index and risky weight control behaviors among White and Native American adolescent girls. Appetite, 51, 210-213.

Madrigal, H., Sánchez-Villegas, A., Martinez-Gonzalez, M. A., Kearney, J., Gibney, M. J., Irala, J., \& Martínez, J. A. (2000). Underestimation of body mass index through perceived body image as compared to self-reported body mass index in the European Union. Public Health, 114, 468-473.

McCabe, M., Ricciardelli, L. A., Sitaram, G., \& Mikhail, K. (2006). Accuracy of body size estimation: Role of biopsychosocial variables. Body Image, 3, 163-171.

Ministério da Saúde (2005). Considerações do ministério da saúde sobre a Pesquisa de Orçamentos Familiares (POF) 2002-2003. Retirado em 16/03/2005, de http://dtr2004.saude.gov.br/nutrição/ redenutri.php.

Monteiro, C. A., Benicio, M. H. D., \& Gouveia, N. C. (2000). Evolução da altura dos brasileiros. Em C. A. Monteiro (Org.), Velhos e novos males da saúde no Brasil (pp. 55-80). São Paulo: Hucitec.

Pinheiro, A. P., \& Giugliani, E. R. (2006). Body dissatisfaction in Brazilian schoolchildren: Prevalence and associated factors. Revista de Saúde Pública, 40, 489-496.

Ramirez E. M., \& Rosen, J. C. (2001). A comparison of weight control plus body image therapy for obese men and women. Journal of Consulting and Clinical Psychology, 69, 440-446.

Ricciardelli, L. A., \& McCabe, M. P. (2001). Children's body image concerns and eating disturbance: A review of the literature. Clinical Psychology Review, 21, 325-344.

Sands, E. R., \& Wardle, J. (2003). Internalization of ideal body shapes in 9-12-year-old girls. International Journal of Eating Disorders, 33, 193-204.

Sanchez-Johnsen, L. A. P., Hogan, K., Wilkens, L. R., \& Fitzgibbon, M. L. (2008). Correlates of problematic eating behaviors in less acculturated Latinas. Eating Behaviors, 9, 181-189.

Skemp-Arlt, K. M., Rees, K. S., Mikat, R. P., \& Seebach, E. E. (2006). Body image dissatisfaction among third, fourth, and fifth grade children. Californian Journal of Health Promotion, 4, 58-67. 
Smolak, L. (2004). Body image in children and adolescents: Where do we go from here? Body Image, 1, 15-28.

Stunkard, A. J., Sorensen, T., \& Schulsinger, F. (1983). Use of the Danish adoption register for the study of obesity and thinness. Em S. Kety, L. P. Rowland, R. L. Sidman \& S. W. Marthysse (Eds.), The genetics of neurological and psychiatric disorders (pp. 115120). New York: Raven.

Stunkard, A. (2000). Old and new scales for the assessment of body image. Perceptual and Motor Skills, 90, 930.

Tehard, B., Van Liere, M. J., Com Nogué, C., \& ClavelChapelon, F. (2002). Anthropometric measurements and body silhouette of women: Validity and perception. Journal of the American Dietetic Association, 102, 1779-1784.

Thompson, J. K. (1996). Body image, eating disorders, and obesity: An integrative guide for assessment and treatment. Washington: American Psychological Association.

Thompson, J. K. (2004). The (mis) measurement of body image: Ten strategies to improve assessment for applied ad research purposes. Body Image, 1, 7-14.

Thompson, M. A., \& Gray, J. J. (1995). Development and validation of a new body image assessment scale. Journal of Personality Assessment, 64, 258-269.
Truby, H., \& Paxton, S. J. (2002). Development of the Children's Body Image Scale. British Journal of Clinical Psychology, 41, 185-203.

Wertheim, E. H., Paxton, S. J., \& Tilgner, L. (2004). Test-retest reliability and construct validity of Countour Drawing Rating Scale scores in a sample of early adolescent girls. Body Image, 1, 199-205.

Williamson, D. A., Womble, L. G., Zucker, N. L., Reas, D. L., White, M. A., Blouin, D. C., \& Greenway, F. (2000). Body image assessment for obesity (BIA-O): Development of a new procedure. International Journal of Obesity, 24, 1326-1332.

Williamson, S., \& Delin, C. (2001). Young children's figural selections: Accuracy of reporting and body size dissatisfaction. International Journal of Eating Disorders, 29, 80-84.

Recebido em 17.02.07 Primeira decisão editorial em 10.10.07 Versão final em 21.03.09 Aceito em 24.03.09 